\title{
THE UNIFORM CONTINUITY OF CONTINUOUS FUNCTIONS ON A TOPOLOGICAL SPACE
}

ALLEN S. DAVIS

Two questions motivate this study: To what extent is it possible to restructure the common domain of a family of continuous functions so that they all become uniformly continuous? When can a group of homeomorphisms of a completely regular space be interpreted as a topological transformation group? It happens that an answer to the first, in the case where range and domain coincide, provides the answer to the second.

The concepts and notation used in this paper, if nonstandard, are defined in [2], with the following exceptions: A structure $V_{I}$ for a set $X$ is called open if for each $x \in X, \alpha \in I$, and $y \in V_{\alpha}(x)$, there is a $\beta \in I$ such that $V_{\beta}(y) \subseteq V_{\alpha}(x) . V_{I}$ is called finite if the space $\left(X, V_{I}\right)$ is totally bounded: For each $\alpha \in I$ there is a finite $S \subseteq X$ such that $X=V_{\alpha}(S)=\bigcup_{x \in S} V_{\alpha}(x)$.

Since every topological space admits a structure [1], the following result implies that in any purely topological study of the family of all functions from one space to another, the notion of continuity can always be replaced by the notion of uniform continuity without loss of generality.

THeORem 1. Given a family $F$ of continuous functions $f: X \rightarrow Y$ from a space $\left(X, U_{I}\right)$ to a space $\left(Y, V_{J}\right)$, there exists a coarsest structure $W_{H}$ as fine as $U_{I}$ such that every member of $F$ is uniformly continuous relative to the pair $\left(W_{H}, V_{J}\right) . U_{I}$ and $W_{H}$ define the same topology for $X$. If both $U_{I}$ and $V_{J}$ are symmetric, open, locally transitive, or transitive, then so is $W_{H}$, respectively. If both $U_{I}$ and $V_{J}$ are finite and $V_{J}$ is uniform, then $W_{H}$ is also finite.

Proof. Let $K$ be the class of all finite subsets of $F$. Define

$$
W_{\alpha a A}=U_{\alpha} \cap \bigcap_{g \in A} g^{-1} V_{a} g,
$$

for $\alpha \in I, a \in J, A \in K$. $W_{I \times J \times K}$ is clearly reflexive and, since

$$
\left[U_{\alpha} \cap U_{\beta}\right] \cap \bigcap_{g \in A \cup B} g^{-1}\left[V_{a} \cap V_{b}\right] g \subseteq W_{\alpha a A} \cap W_{\beta b B}
$$

it is a base for a structure $W_{H}$. This structure is obviously as fine as $U_{I}$. On the other hand, $U_{I}$ is locally as fine as $W_{H}$ : Given $x \in X$ and

Received by the editors July 23, 1962. 
$(\alpha, a, A) \in I \times J \times K$, it is possible to choose $\beta \in I$ so that $U_{\beta} \subseteq U_{\alpha}$ and so that $U_{\beta}(x) \subseteq g^{-1} V_{a} g(x)$, for all $g \in A$, because $A$ is a finite set of continuous functions and $U_{I}$ is closed under intersection. Then $U_{\beta}(x) \subseteq W_{\alpha a A}(x)$. Thus $U_{I}$ and $W_{H}$ define the same topology. Now for each $f \in F$ and each $a \in J, W_{\alpha a\{f\}} \subseteq f^{-1} V_{a} f$, for any $\alpha \in I$, whence, $f$ is uniformly continuous $r e\left(W_{H}, V_{J}\right)$. Let us show that $W_{H}$ is as coarse as possible. Suppose that each member of $F$ is uniformly continuous re $\left(W_{H^{\prime}}^{\prime}, V_{J}\right)$, for some $W_{H^{\prime}}^{\prime}$ as fine as $U_{I}$. Given $(\alpha, a, A)$ $\in I \times J \times K$, choose $\alpha^{\prime} \in H^{\prime}$ so that $W_{\alpha^{\prime}}^{\prime} \subseteq g^{-1} V_{a} g$, for all $g \in A$, and so that $W_{\alpha^{\prime}}^{\prime} \subseteq U_{\alpha}$. Then $W_{\alpha^{\prime}}^{\prime} \subseteq W_{\alpha a A}$. Thus $W_{H^{\prime}}^{\prime}$ is as fine as $W_{H}$. It is also straightforward to show that the various properties possessed by $U_{I}$ and $V_{J}$ are inherited (as recessive traits) by $W_{H}$. For example, suppose both $U_{I}$ and $V_{J}$ are locally transitive. Given $x \in X$ and $(\alpha, a, A) \in I \times J \times K$, choose $\beta \in I$ so that $U_{\beta}^{2}(x) \subseteq U_{\alpha}(x)$ and choose, for each $g \in A, b(g) \in J$ so that $V_{b(o)}^{2}(g(x)) \subseteq V_{\alpha}(g(x))$. Then let $V_{b}=\bigcap_{g \in A} V_{b(g)}$. We have by a simple calculation with dyadic relations, $W_{\beta b A}(x) \subseteq U_{\beta}^{2}(x) \cap \bigcap_{o \in A} g^{-1} V^{2} g(x) \subseteq W_{\alpha a A}(x)$, whence, $W_{H}$ is also locally transitive. Finally, consider the hypothesis that $U_{I}$ and $V_{J}$ be finite. There is no difficulty in showing that subspaces of totally bounded uniform spaces are totally bounded, so if $V_{J}$ is uniform, then the range of each member of $F$ is totally bounded. Given $(\alpha, a, A)$ $\in I \times J \times K$, we shall find a finite subset $S \subseteq X$ such that $X=W_{\alpha a A}(S)$. Let $S_{0} \subseteq X$ be a finite subset for which $X=U_{\alpha}\left(S_{0}\right)$ and, for each $g \in A$, let $T_{g} \subseteq g(X)$ be a finite subset for which $g(X) \subseteq V_{a}\left(T_{g}\right)$. Construct $S_{\theta} \subseteq X$ by choosing an $s \in g^{-1}(y)$, for each $y \in T_{g}$. Then it is easy to see that $S=S_{0} \cup \cup_{g \in A} S_{g}$ has the desired properties. Therefore $W_{H}$ is also finite.

Query. Are there subspaces of totally bounded spaces with symmetric locally transitive structures which are not totally bounded?

Corollary (Levine). If $U_{I}$ and $V_{J}$ are equivalent to metric structures and $F$ is countable, then $W_{H}$ is equivalent to a metric structure.

Proof. A (Hausdorff) structure is equivalent to a metric structure if and only if it is uniform and has a countable base [6]. If $F$ is countable and countable bases are involved in the construction of $W_{H}$, then $W_{H}$ receives a countable base.

Note. In [3] an appropriate metric for $X$ is given explicitly in terms of $F$ and the original metrics for $X$ and $Y$. In fact, if the original metrics are $\delta_{1}$ and $\delta_{2}$, respectively, and $\delta_{1}^{*}$ is defined on $X \times X$ by

$$
\delta_{1}^{*}(p, q)=\delta_{1}(p, q)+\sum_{i=1}^{\infty} 2^{-i} \min \left[1, \delta_{2}\left(f_{i}(p), f_{i}(q)\right)\right]
$$


where $F=\left\{f_{1}, f_{2}, \cdots\right\}$, then $\delta_{1}$ and $\delta_{1}^{*}$ define the same topology for $X$ and every member of $F$ is $\left(\delta_{1}^{*}, \delta_{2}\right)$-uniformly continuous. Note that $\delta_{2}$ need not be changed. (See also [4].)

TheOREm 2. Given a space $\left(X, U_{I}\right)$ and a family $F$ of continuous transformations $f: X \rightarrow X$, there is a coarsest structure $W_{H}$ as fine as $U_{I}$, relative to which every member of $F$ is uniformly continuous. $U_{I}$ and $W_{H}$ define the same topology for $X$ and $W_{H}$ inherits as in Theorem 1 mutatis mutandis the structure properties possessed by $U_{I}$.

Proof. Let $F^{*}$ be the semigroup generated by $F$ under composition, plus the identity $\Delta: X \rightarrow X: x \rightarrow x$. Let $K$ be the class of all finite subsets of $F^{*}$ containing $\Delta$. Define

$$
W_{\alpha A}=\bigcap_{0 \in A} g^{-1} U_{\alpha} g
$$

for $\alpha \in I, A \in K$. The proof then proceeds very much like that of Theorem 1, except that we show uniform continuity as follows: Given $f \in F$ and $(\alpha, A) \in I \times K$, let $B=\{g f\}_{g \in A} \cup\{\Delta\}$. Then $B \in K$ and $W_{\alpha B}=U_{\alpha} \cap \bigcap_{g \in A} f^{-1} g^{-1} U_{\alpha} g f \subseteq f^{-1} W_{\alpha A} f$, so that $f$ is uniformly continuous re $W_{H}$.

COROLLARY. Every group of homeomorphisms of a completely regular space is a topological homeomorphism group. That is, there exists an admissible topology relative to which composition and inversion are continuous.

Proof. Let the topology of the given completely regular space be defined by a uniform structure $V_{I}$ relative to which every continuous $f: X \rightarrow X$ is uniformly continuous. The existence of such a $V_{I}$ follows from a well-known theorem by Weil [6] together with Theorem 2 . Define for the set $F$ of homeomorphisms in question the uniform structure $V_{I}^{*}$ by putting $V_{\alpha}^{*}(f)=\left\{g \in F: g \subseteq V_{\alpha} f\right\}(\alpha \in I, f \in F)$. This provides $F$ with the "topology of uniform convergence," with respect to which the map $F \times X \rightarrow X:(f, x) \rightarrow f(x)$ is continuous, as is well known. The continuity of $\Phi: F \times F \rightarrow F:(f, g) \rightarrow f^{-1} g$ follows from a theorem proved by Ford [5] together with the uniform continuity of the members of $F$. In fact, given $f, g \in F$ and $\alpha \in I$, choose $\beta \in I$ so that $V_{\beta}^{2} \subseteq V_{\alpha}$ and $\gamma \in I$ so that $V_{\gamma}$ is symmetric and $f^{-1} V_{\gamma} \subseteq V_{\beta} f^{-1}$. Then $\Phi$ maps $V_{\gamma}^{*}(f) \times V_{\gamma}^{*}(g)$ into $V_{\alpha}^{*}\left(f^{-1} g\right)$.

Note. There is also a corollary to Theorem 2 similar to the one following Theorem 1. In fact, if $F=\left\{f_{1}, f_{2}, \cdots\right\}$ is a countable family of continuous transformations of a metric space $(X, \delta)$, then the function $\delta^{*}: X \times X \rightarrow[0, \infty)$ defined by 
$\delta^{*}(p, q)=\delta(p, q)+\sum_{r=1}^{\infty} \sum_{\kappa \in S_{r}}\left(\frac{1}{2}\right)^{k_{1}+\cdots+k_{r}} \delta^{\prime}\left(f_{k_{1}} \cdots f_{k_{r}}(p), f_{k_{1}} \cdots f_{k_{r}}(q)\right)$, where $\delta^{\prime}=\min [1, \delta]$ and $S_{i}$ is the set of all $r$-tuples $\kappa=\left(k_{1}, \cdots, k_{r}\right)$ of natural numbers, can be seen to be a metric defining the same topology as $\delta$ and such that, for every $k=1,2, \cdots$, and all $p, q \in X$, $\delta^{*}\left(f_{k}(p), f_{k}(q)\right) \leqq 2^{k} \delta^{*}(p, q)$. (Cf. [4].)

\section{REFERENCES}

1. A. S. Davis, Indexed systems of neighborhoods for general topological spaces, Amer. Math. Monthly 68 (1961), 886-893.

2. - Fixpoint theorem for contractions of a well-chained topological space, Proc. Amer. Math. Soc. 14 (1963), 981-985.

3. N. Levine, Remarks on uniform continuity in metric spaces, Amer. Math. Monthly 67 (1960), 562-563.

4. $-A$ note on the Lipschitz condition in metric spaces, Proc. Amer. Math. Soc. 13 (1962), 314-315.

5. L. R. Ford, Homeomorphism groups and coset spaces, Trans. Amer. Math. Soc. 77 (1954), 490-497.

6. A. Weil, Sur les espaces à structure uniforme et sur la topologie générale, Actualités Sci. Ind. No. 551, Paris, Hermann, 1937.

UNIVERSITY OF OKLAHOMA 